\title{
Structurally investigating a niche pathway for chemical reversal of proline hydroxylation in the pathogen C. difficile \\ L Backman ${ }^{1}$ \\ ${ }^{1}$ Massachusetts Institute of Technology \\ Ibackma2@mit.edu
}

The glycyl radical enzyme (GRE) family utilizes a glycyl radical cofactor, installed by AdoMet radical activating enzymes, to catalyze difficult chemical reactions in a variety of microbial metabolic pathways. Although glycyl radical enzymes are widely encoded and expressed by bacteria found in the gut microbiome, these enzymes remain largely uncharacterized. Recently, a new glycyl radical enzyme was discovered to catalyze the dehydration of trans4-hydroxy-L-proline (4-Hyp) to 1-pyrroline-5-carboxylic acid. Bioinformatics studies by the Balskus lab show that this hydroxyproline dehydratase (HypD) is the second most prominent GRE in the human gut microbiome and is encoded by 360 bacterial genomes, including the human pathogen C. difficile. HypD presents a pathway for bacteria to reverse 4-Hyp post-translational modifications, the most common post-translational modification in animals which was previously thought to be irreversible. Furthermore, the bacteria that encode HypD are known to use 4Hyp as an electron acceptor during amino acid fermentation, their primary method of generating adenosine triphosphate (ATP). However, the enzyme responsible for assimilating 4-Hyp into this pathway has remained unknown until now. HypD could be the missing puzzle piece to understanding how these bacteria use the abundant metabolite 4-Hyp in energy production, while also symbiotically providing humans with a method for recycling this common amino acid. In order to elucidate the mechanism for how HypD performs the dehydration of hydroxyproline, we aimed to characterize HypD from C. difficile, in the presence of its substrate. Here, we have solved a $2.05-\AA$ resolution structure for HypD by molecular replacement. Subsequently, a structure for HypD with its substrate 4-Hyp bound in the active site was solved to $2.52-\AA \AA$ resolution. These structures and accompanying biochemical studies have led us to identify key catalytic residues and have provided insight into the mechanism for 4-Hyp dehydration.

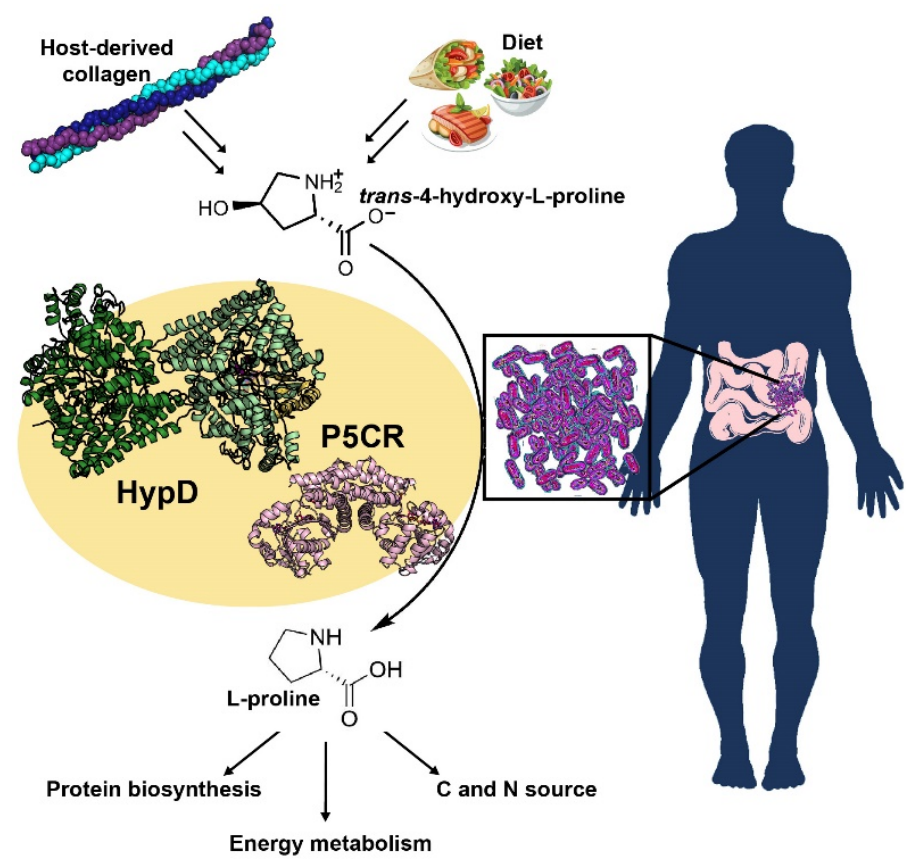

Figure 1 